\title{
CONVERGING FACTORS FOR SOME ASYMPTOTIC MOMENT SERIES THAT ARISE IN NUMERICAL QUADRATURE
}

\author{
AVRAM SIDI ${ }^{1}$
}

(Received 25 August 1980; revised 22 December 1981)

\begin{abstract}
In this work the asymptotic behavior of the partial sums of the divergent asymptotic moment series $\sum_{t=1}^{\infty} \mu_{t} / z^{t}$, where $\mu_{1}$ are the moments of the weight functions $w(x)=x^{\alpha} e^{-x}$, $\alpha>-1$, and $w(x)=x^{\alpha} E_{m}(x), \alpha>-1, m+\alpha>0$, on the interval $[0, \infty)$, is analyzed. Expressions for the converging factors are derived. These converging factors form the basis of some very accurate numerical quadrature formulas derived by the author for the infinite range integrals $\int_{0}^{\infty} w(x) f(x) d x$ with $w(x)$ as given above.
\end{abstract}

\section{Introduction}

Recently a new approach to numerical quadrature has been presented by the author, see [5]. In this approach numerical quadrature formulae $I_{k}[u]$ for the integral $I[u]$, where

$$
\begin{aligned}
& I[u]=\int_{a}^{b} w(x) u(x) d x, \\
& I_{k}[u]=\sum_{i=1}^{k} A_{k l} u\left(x_{k \imath}\right),
\end{aligned}
$$

are derived by forming a sequence of rational approximations $H_{k}(z), k=1,2, \ldots$, to the function $H(z)$, where

$$
\begin{aligned}
& H(z)=\int_{a}^{b} \frac{w(x)}{z-x} d x, \\
& H_{k}(z)=\sum_{i=1}^{k} \frac{A_{k i}}{z-x_{k i}} .
\end{aligned}
$$

\footnotetext{
' Computer Science Department, Technion-Israel Institute of Technology, Haifa, Israel. (c) Copyright Australian Mathematical Society 1982
} 
The function $H(z)$ is analytic in the complex $z$-plane cut along the interval $[a, b]$.

We shall not deal with the motivation of this approach in this work, as that has been done in detail in [5]. We shall only state that from the motivation in the work above one could conclude heuristically that $I_{k}[u]$ would be a good approximation to $I[u]$ if $H_{k}(z)$ is a good approximation to $H(z)$ in the complex $z$-plane cut along $[a, b]$, and that $I_{k}[u] \rightarrow I[u]$ quickly as $k \rightarrow \infty$ if $H_{k}(z) \rightarrow H(z)$ quickly as $k \rightarrow \infty$ in the complex $z$-plane cut along $[a, b]$.

The rational approximations $H_{k}(z)$ in [5] are obtained by applying some modified version of the $T$-transformation of [1] to the moment series of $H(z)$, namely to the series

$$
H(z) \sim \sum_{i=1}^{\infty} \frac{\mu_{i}}{z^{i}}, \quad \text { as } z \rightarrow \infty, z \notin[a, b],
$$

where $\mu_{1}$ are the moments of $w(x)$,

$$
\mu_{i}=\int_{a}^{b} w(x) x^{i-1} d x, \quad i=1,2, \ldots
$$

It can easily be seen that if $[a, b]$ is finite, then the series (1.3) converges for $|z|>\max (|a|,|b|)$. If, however, $[a, b]$ is infinite, like $[0, \infty)$, then $(1.3)$ is a divergent asymptotic series.

As explained in [5] (see also [3] and [4], in which convergence properties of the $T$-transformation are analyzed), the $T$-transformation, when applied to the sequence of the partial sums of the infinite series in (1.3), produces very good approximations to $H(z)$, provided that

$$
H(z)=\sum_{i=1}^{n-1} \frac{\mu_{t}}{z^{i}}+R_{n} f(n)
$$

where $R_{n}$ are numbers related to the moments, and $f(y)$; as a function of the continuous variable $y$, has an asymptotic expansion of the form

$$
f(y) \sim \sum_{i=0}^{\infty} \frac{\beta_{t}}{y^{i}} \text { as } y \rightarrow \infty,
$$

and is infinitely differentiable up to $y=\infty$. We notice that the term $R_{n} f(n)$ in (1.5) serves as a "converging factor" for the series in (1.3).

For $a=0, b=1$, and $w(x)=(1-x)^{\alpha} x^{\beta}(-\log x)^{\nu}, \alpha+\nu>-1, \beta>-1$, it has been shown in [5] that (1.5) holds with $R_{n}=1 /\left(n^{\alpha+\nu+1} z^{n}\right)$ and

$$
f(y)=y^{\alpha+\nu+1} \int_{0}^{\infty} e^{-y t} t^{\alpha+\nu} g(t) d t,
$$

where

$$
g(t)=\left[\left(1-e^{-t}\right) / t\right]^{\alpha} e^{-\beta t} /\left(1-e^{-t} / z\right)=\sum_{t=0}^{\infty} g_{t} t^{t}
$$


hence (1.6) is also satisfied with $f(y) \sim \sum_{i=0}^{\infty} g_{i}(\alpha+\nu+i) ! / y^{t}$ as $y \rightarrow \infty$, for all $z \notin[0,1]$. (Actually $R_{n}, f(n), g(t)$, and $g_{l}$ should be written as $R_{n}(z), f(n, z)$, $g(t, z)$, and $g_{i}(z)$. However, $z$ is fixed and we do not perform any operation with respect to $z$. Hence we omit $z$ without fear of confusion.) This expansion can be obtained by applying Watson's lemma, see [2, page 71], to the integral representation of $f(y)$. Since (1.5) and (1.6) are satisfied, the numerical quadrature formulae for this $w(x)$ turn out to be very accurate. For more details and numerical results see [5].

We note that $f(y) / y$ is the Laplace transform of a function $\varphi(t)$ which is analytic at $t=0$ and has a Maclaurin series with a finite radius of convergence (that of the Maclaurin series of $g(t)$ ). This follows from a more general result which is given in the appendix to the present work.

In a recent work, [6], very accurate numerical quadrature formulae for infinite range integrals with $a=0, b=\infty$, and weight functions $w(x)=x^{\alpha} e^{-x}, \alpha>-1$, and $w(x)=x^{\alpha} E_{m}(x), \alpha>-1, m+\alpha>0$, where $E_{m}(x)$ is the exponential integral, have been developed using the approach of [5]. These formulae are based on the results of Section 2 and Section 3 of the present work, which show that also in these cases the functions $H(z)$ satisfy (1.5) and (1.6). Furthermore, for $\operatorname{Re} z<0$, it is shown that $f(y) / y$ for these cases are Laplace transforms of entire functions $\varphi(t)$, which, we believe, should be of some importance in the convergence analysis of $H_{k}(z)$ to $H(z)$.

2. The case $w(x)=x^{\alpha} e^{-x}, \alpha>-1$

Let $w(x)=x^{\alpha} e^{-x}, \alpha>-1$. Then from (1.4) we have

$$
\mu_{i}=\int_{0}^{\infty} e^{-x} x^{\alpha+i-1} d x=(\alpha+i-1) !, \quad i=1,2, \ldots
$$

By substituting the relation

$$
\frac{1}{z-x}=\sum_{i=1}^{n-1} \frac{x^{i-1}}{z^{i}}+\frac{1}{z^{n}} \frac{x^{n-1}}{1-x / z}
$$

and (2.1) in (1.2), we have

$$
H(z)=\int_{0}^{\infty} \frac{x^{\alpha} e^{-x}}{z-x} d x=\sum_{i=1}^{n-1} \frac{\mu_{i}}{z^{i}}+\frac{J(\alpha+n-1, z)}{z^{n}},
$$

where

$$
J(p, z)=\int_{0}^{\infty} \frac{e^{-x} x^{p}}{1-x / z} d x
$$

which is analytic for all $z$ not on the positive real axis. 
All we have to analyze then is $J(p, z)$. Now for $\operatorname{Re} z<0$, we have $\operatorname{Re}\left[e^{\operatorname{targ}(-z)}(1-x / z)\right]>0$ whenever $0 \leqslant x<\infty$. It then follows that

$$
\frac{e^{-x}}{1-x / z}=e^{-z} \int_{-z}^{\infty} e^{-\tau(1-x / z)} d \tau
$$

where the integral is taken along the straight line path $L$ in the $\tau$-plane, which starts at $\tau=-z$ and extends to $\tau=\infty$, and along which $\arg \tau=\arg (-z)$, see Figure 1. Substituting (2.5) in (2.4), and changing the order of integration, we have

$$
J(p, z)=e^{-z} \int_{-z}^{\infty} d \tau e^{-\tau} \int_{0}^{\infty} d x e^{-(-\tau / z) x} x^{p}
$$

$(L)$

which, upon using the fact that

$$
\int_{0}^{\infty} e^{-t x} x^{q} d x=\frac{q !}{t^{q+1}}, \quad q>-1, \quad \operatorname{Re} t>0,
$$

becomes

$$
J(p, z)=e^{-z} p !(-z)^{p_{*}^{+1}} \int_{-z}^{\infty} d \tau \frac{e^{-\tau}}{\tau^{p+1}}
$$

where $\tau^{s}$, for $s$ real, is taken to be positive real for $\tau$ positive real, with its branch cut along the negative real axis, i.e., $\tau^{s}=|\tau|^{s} e^{i s \arg (\tau)},|\arg (\tau)|<\pi$, similarly for $(-z)^{s}$. The change of order of integration in (2.6) is allowed since the integrand is absolutely integrable both at $x=\infty$ and $\tau=\infty e^{i \arg (-z)}$.

Now (2.8) can also be expressed as

$$
J(p, z)=e^{-z} p !(-z)^{p+1} \int_{-z}^{\infty} d \tau \frac{e^{-\tau}}{\tau^{p+1}},
$$

where the integral this time is taken along the contour $\Gamma$ that starts at $\tau=-z$ and approaches the real $\tau$-axis asymptotically, see Figure 1 . Since the integral in (2.9)

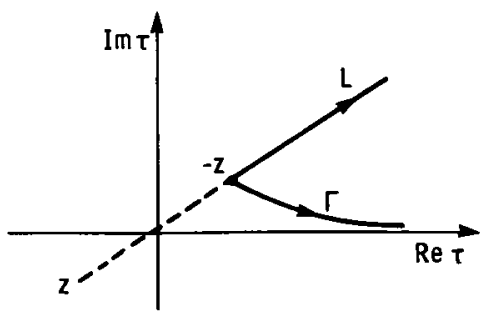

Figure 1. The contours $\Gamma$ and $L$ in the $\tau$-plane for the case $\operatorname{Re} z<0$. 
converges both when $\operatorname{Re} z<0$ and $\operatorname{Re} z \geqslant 0$, by analytic continuation, $J(p, z)$ is given by (2.9) for all $z$, such that $z \notin[0, \infty)$.

LEMMA 1. Let $C$ be a contour in the complex plane that extends from $\zeta$ to infinity and define

$$
M[F ; \zeta]=\int_{\zeta}^{\infty} \frac{F(\tau)}{\tau^{q+1}} d \tau
$$

Assume that along $C$ the function $F(\tau)$ is infinitely differentiable and that $\left(S^{\prime} F\right)(\tau)$ $=O\left(\tau^{q}\right)$ as $\tau \rightarrow \infty$ along $C$, where the operators $S^{\boldsymbol{I}}$ are defined as follows: $(S F)(\tau)=\tau F^{\prime}(\tau), S^{0} F=F, S^{i} F=S\left(S^{i-1} F\right), i=1,2, \ldots$ Then for any positive integer $N$,

$$
M[F ; \zeta]=\frac{1}{\zeta^{q}} \sum_{i=0}^{N-1} \frac{\left(S^{\imath} F\right)(\zeta)}{q^{i+1}}+\frac{1}{q^{N}} M\left[S^{N} F ; \zeta\right] .
$$

Proof. Integrating $(2.10)$ by parts, we obtain

$$
M[F ; \zeta]=\frac{1}{\zeta^{q}} \frac{F(\zeta)}{q}+\frac{1}{q} M[S F ; \zeta] .
$$

Equation (2.11) now follows by repeated application of (2.12) to $S^{\prime} F, i=$ $1,2, \ldots, N-1$.

COROLlary 2. Let the contour $C$ be such that

$$
\int_{\zeta}^{\infty} \frac{|d \tau|}{|\tau|^{q+1}}=O\left(|\zeta|^{-q}\right) \quad \text { as } q \rightarrow \infty,
$$

and let $\left(S^{i} F\right)(\tau)=o(1)$ as $\tau \rightarrow \infty$ along $C, i=0,1,2, \ldots$. Then $M[F ; \zeta]$, as $q \rightarrow \infty$, has an asymptotic expansion given by

$$
M[F ; \zeta] \sim \frac{1}{\zeta^{q}} \sum_{i=0}^{\infty} \frac{\left(S^{i} F\right)(\zeta)}{q^{i+1}} .
$$

Proof. It is enough to show that in (2.11)

$$
\zeta^{q} M\left[S^{N} F ; \zeta\right]=O\left(q^{-1}\right) \text { as } q \rightarrow \infty .
$$

Now from (2.12)

$$
\zeta^{q} M\left[S^{N} F ; \zeta\right]=\frac{\left(S^{N} F\right)(\zeta)}{q}+\frac{1}{q} \zeta^{q} M\left[S^{N+1} F ; \zeta\right] .
$$

Since $\left(S^{i} F\right)(\tau)=o(1)$ as $\tau \rightarrow \infty$ along $C$ for any $i$, there exist finite positive constants $\lambda_{i}$ such that $\lambda_{t}=\max _{\tau \in C}\left|\left(S^{i} F\right)(\tau)\right|, i=0,1, \ldots$. Substituting in 
(2.16) the integral representation of $M\left[S^{N+1} F ; \zeta\right]$ and taking the modulus of both sides, we obtain

$$
\left|\zeta^{q} M\left[S^{N} F ; \zeta\right]\right| \leqslant \frac{\lambda_{N}}{q}+\frac{\lambda_{N+1}}{q}|\zeta|^{q} \int_{\zeta}^{\infty} \frac{|d \tau|}{|\tau|^{q+1}} .
$$

Using now (2.13) in (2.17), the result follows.

REMARK 3. Parametric representations for two types of contours $C$, for which (2.13) is valid, are given below:

(Type 1) $\tau=\zeta r$, r real, $1 \leqslant r<\infty$,

(Type 2) $C=C_{1} \cup C_{2}$, where $C_{1}$ is defined parametrically by $\tau=\zeta e^{i \theta}$, $\min \left(0, \theta_{0}\right) \leqslant \theta \leqslant \max \left(0, \theta_{0}\right)$ for some fixed $\theta_{0}$ such that $0<\left|\theta_{0}\right|<\pi$, and $C_{2}$ is defined parametrically by $\tau=\zeta \mathrm{re}^{i \theta_{0}}, r$ real, $1 \leqslant r<\infty$. For contours of Type 1

$$
\int_{\zeta(C)}^{\infty} \frac{|d \tau|}{|\tau|^{q+1}}=\frac{1}{|\zeta|^{q}}
$$

whereas for those of Type 2

$$
\int_{\zeta(C)}^{\infty} \frac{|d \tau|}{|\tau|^{q+1}}=\frac{\left|\theta_{0}\right|}{|\zeta|^{q}}+\frac{1}{q|\zeta|^{q}}
$$

Note that the contour $L$ in Figure 1 is a contour of Type 1 with $\zeta=-z$.

Going back to (2.9), we can see that the above lemma and its corollary can be applied quite easily to the integral on the right hand side by letting $F(\tau)=e^{-\tau}$, $q=p$, and $\zeta=-z$. Since the integrand is analytic everywhere except on the branch cut along the negative real axis, the contour $\Gamma$ can be deformed to a contour of Type 2 as in Figure 2.

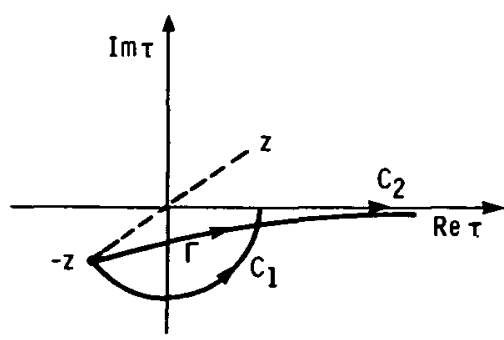

Figure 2. Deformation of $\Gamma$ to a contour of Type 2. 
Applying now Lemma 1 and Corollary 2 to (2.9), we obtain

$$
J(p, z) \sim-z e^{-z} p ! \sum_{i=0}^{\infty} \frac{\gamma_{i}(z)}{p^{i+1}} \quad \text { as } p \rightarrow \infty,
$$

where

$$
\gamma_{i}(z)=\left.\left(S^{i} F\right)(\tau)\right|_{\tau=-z}=\left(z \frac{d}{d z}\right)^{i} e^{z}, \quad i=0,1,2, \ldots
$$

Using the Maclaurin series expansion of $e^{z}, \gamma_{l}(z)$ can be expressed by the infinite series

$$
\gamma_{i}(z)=\sum_{k=0}^{\infty} \frac{k^{i}}{k !} z^{k}, \quad i=0,1,2, \ldots
$$

As explained in the Introduction, what goes into the $T$-transformation is a converging factor in terms of an infinite series in inverse powers of $y$. Such an expansion can also be found quite easily. Letting $p=\alpha+y-1$, we can express (2.9) in the form

$$
J(\alpha+y-1, z)=e^{-z}(\alpha+y-1) !(-z)^{\alpha+y-1} \int_{-z}^{\infty} d \tau \frac{\left(e^{-\tau} / \tau^{\alpha-1}\right)}{\tau^{y+1}}
$$

Applying now Lemma 1 and Corollary 2 with $F(\tau)=e^{-\tau} / \tau^{\alpha-1}, q=y$ and $\zeta=-z$, with the contour $\Gamma$ deformed as above, we obtain

$$
J(\alpha+y-1, z) \sim e^{-z}(\alpha+y-1) !(-z)^{\alpha-1} \sum_{i=0}^{\infty} \frac{\varepsilon_{t}(z)}{y^{t+1}} \text { as } y \rightarrow \infty,
$$

where

$$
\varepsilon_{i}(z)=\left.\left(\tau \frac{d}{d \tau}\right)^{\prime} \frac{e^{-\tau}}{\tau^{\alpha-1}}\right|_{\tau=-z}, \quad i=0,1,2, \ldots
$$

We can therefore conclude that (1.5) and (1.6) are satisfied with

$$
R_{n}=\frac{(\alpha+n-1) !}{n z^{n}}=\frac{\mu_{n} / z^{n}}{n}
$$

and

$$
f(y) \sim(-z)^{\alpha-1} e^{-z} \sum_{t=0}^{\infty} \frac{\varepsilon_{t}(z)}{y^{i}} \quad \text { as } y \rightarrow \infty .
$$

REMARK 4. When Levin's t-transformation is applied to the sequence of the partial sums of the series (1.3), one takes $R_{n}=\mu_{n} / z^{n}$. From (2.18) we see that $R_{n}$ should actually be $\left(\mu_{n} / z^{n}\right) n^{-1}$. However, the results of using either $R_{n}$ in the T-transformation are about the same, see [5]. 
We now wish to show that the function $f(y)$ can be expressed in terms of a Laplace transform of an entire function whenever $\operatorname{Re} z<0$. Making the change of variable $\tau=-z e^{\xi}$ in the integral in (2.8), we obtain

$$
J(p, z)=-z e^{-z} p ! \int_{0}^{\infty} e^{-p \xi} \exp \left(z e^{\xi}\right) d \xi
$$

or

$$
J(\alpha+y-1, z)=-z e^{-z}(\alpha+y-1) ! \int_{0}^{\infty} e^{-y \xi} h(\xi, z) d \xi,
$$

where $h(\xi, z)=e^{(1-\alpha) \xi} \exp \left(z e^{\xi}\right)$ is an entire function of $\xi$. Hence by (1.5) and (2.24)

$$
f(y)=-z e^{-z} y \int_{0}^{\infty} e^{-y \xi} h(\xi, z) d \xi,
$$

which is a Laplace transform. Using Watson's lemma in (2.26) (or (2.27)), the existence of the asymptotic expansions in (2.18) (or (2.22)) and the expressions given in (2.20) and (2.23) can be re-established.

3. The case $w(x)=x^{\alpha} E_{m}(x), \alpha>1, m+\alpha>0$

Let $w(x)=x^{\alpha} E_{m}(x)$, where $E_{m}(x)=\int_{1}^{\infty}\left(e^{-x t} / t^{m}\right) d t$. Then for $\alpha>-1$ and $m+\alpha>0$

$$
\mu_{t}=\int_{0}^{\infty} d x x^{\alpha+t-1} \int_{1}^{\infty} d t \frac{e^{-x t}}{t^{m}},
$$

which, upon changing the order of integration and using (2.7), becomes

$$
\mu_{i}=\frac{(\alpha+i-1) !}{m+\alpha+i-1}, \quad i=1,2, \ldots
$$

Substituting again (2.2) in (1.2), and using (3.2), we obtain

$$
H(z)=\int_{0}^{\infty} \frac{x^{\alpha} E_{m}(x)}{z-x} d x=\sum_{i=1}^{n-1} \frac{\mu_{i}}{z^{i}}+\frac{K(\alpha+n-1, z)}{z^{n}},
$$

where

$$
K(p, z)=\int_{0}^{\infty} \frac{x^{p} E_{m}(x)}{1-x / z} d x=\int_{0}^{\infty} d x \frac{x^{p}}{1-x / z} \int_{1}^{\infty} d t \frac{e^{-x t}}{t^{m}},
$$

which, upon changing the order of integration and then making the change of variable $x t=u$ in the integral with respect to $x$ ( $t$ fixed), is seen to be

$$
K(p, z)=\int_{1}^{\infty} \frac{d t}{t^{m+p+1}} J(p, t z),
$$

where $J(p, z)$ is as defined in the previous section. 
We now consider the case $\operatorname{Re} z<0$. Since in (3.5) $1 \leqslant t<\infty$, for this case $\operatorname{Re}(t z)<0$ too. Hence we can make use of the integral representation for $J(p, z)$ given in (2.26), with $z$ replaced by $t z$. We then obtain

$$
K(p, z)=-z p ! \int_{1}^{\infty} \frac{d t}{t^{m+p}} e^{-t z} \int_{0}^{\infty} d \xi e^{-p \xi} \exp \left(t z e^{\xi}\right) .
$$

Making the change of variable $t=e^{\sigma}$ in (3.6), we have

$$
K(p, z)=-z p ! \int_{0}^{\infty} d \sigma e^{-(m+p-1) \sigma} \exp \left(-z e^{\sigma}\right) \int_{0}^{\infty} d \xi e^{-p \xi} \exp \left(z e^{\xi+\sigma}\right) .
$$

Making the further change of variables $(\xi, \sigma) \rightarrow\left(\omega, \sigma^{\prime}\right)$, where $\omega=\xi+\sigma$, and $\sigma^{\prime}=\sigma$, we can express (3.7) as

$$
K(p, z)=-z p ! \int_{0}^{\infty} d \omega e^{-p \omega} \exp \left(z e^{\omega}\right) B(\omega, z),
$$

where

$$
B(\omega, z)=\int_{0}^{\omega} d \sigma e^{(1-m) \sigma} \exp \left(-z e^{\sigma}\right) .
$$

Note that $B(\omega, z)$ is an entire function of $\omega$. Letting $p=\alpha+y-1$, and writing the integrand in equation (3.8) in the form $e^{-y \omega} A(\omega, z)$, it is very easy to see that $A(\omega, z)$ is an entire function of $\omega$, hence

$$
K(\alpha+y-1, z) \sim \frac{(\alpha+y-1) !}{y} \sum_{i=0}^{\infty} \frac{-z \varepsilon_{t}(z)}{y^{\prime}} \text { as } y \rightarrow \infty,
$$

where

$$
\varepsilon_{i}(z)=\partial^{\prime} A(\omega, z) /\left.\partial \omega^{i}\right|_{\omega=0}, \quad i=0,1, \ldots
$$

A similar result for the case $\operatorname{Re} z \geqslant 0$ could probably be obtained, however this seems to be rather complicated and shall not be pursued further in this work.

We have shown that, at least for $\operatorname{Re} z<0, H(z)$ satisfies (1.5) with (1.6), where $R_{n}=\left[(\alpha+n-1) ! / z^{n}\right] n^{-1}$, such that the expansion $f(y) / y$ is the Laplace transform of an entire function. Note that this $R_{n}$ is independent of $m$ and is the same as that obtained for $w(x)=x^{\alpha} e^{-x}, \alpha>-1$, whereas in the $t$-transformation of Levin $R_{n}=\mu_{n} / z^{n}$, hence this $R_{n}$ depends on $m$ through $\mu_{n}=(\alpha+n-$ $1) ! /(m+\alpha+n-1)$. These observations have some important implications in the development of the new numerical quadrature formulae as explained in [6].

\section{Appendix}

The Laplace transform $\bar{u}(p)$ of a function $u(t)$ is defined by

$$
\bar{u}(p)=\mathscr{L}[u(t) ; p]=\int_{0}^{\infty} e^{-p t} u(t) d t .
$$


THEOREM. Let $g(t)$ be an analytic function at $t=0$ and let its Maclaurin series

$$
g(t)=\sum_{i=0}^{\infty} g_{i} t^{t}
$$

have radius of convergence $\rho$. Define

$$
G(p)=\mathscr{L}\left[t^{\circ} g(t) ; p\right], \quad \sigma>-1 .
$$

Then there exists a function $h(t)$, which is analytic at $t=0$ and has a Maclaurin series with radius of convergence $\rho$, such that

$$
\bar{h}(p)=p^{\circ} G(p) .
$$

Actually for $|t|<\rho$

$$
h(t)=\sum_{i=0}^{\infty} \frac{(\sigma+i) !}{i !} g_{t} t^{t}
$$

Proof. We shall deal with the case $-1<\sigma<0$ first. Since $-\sigma-1>-1$, $p^{\sigma}=\mathcal{E}\left[t^{-\sigma-1} /(-\sigma-1) ! ; p\right]$. Hence $p^{\sigma} G(p)$ is the Laplace transform of the convolution

$$
h(t)=\int_{0}^{t} \frac{(t-\tau)^{-\sigma-1}}{(-\sigma-1) !} \tau^{\sigma} g(\tau) d \tau .
$$

Let $t \leqslant \bar{\rho}<\rho$. Then substituting (A.2) in (A.6), and changing the order of summation and integration, we obtain

$$
h(t)=\sum_{i=0}^{\infty} g_{i} \int_{0}^{t} \frac{(t-\tau)^{-\sigma-1}}{(-\sigma-1) !} \tau^{\sigma+i} d \tau .
$$

This is allowed since the Maclaurin series of $g(t)$ converges absolutely and uniformly for $|t| \leqslant \bar{\rho}<\rho$. Using the fact that the integral in the summation is a convolution, (A.S) is easily obtained. Using the ratio test, the infinite power series in (A.5) can be shown to have the same radius of convergence as that of (A.2), namely $\rho$. This completes the proof for the case $-1<\sigma<0$.

If we let $\sigma=0$, then (A.4) and (A.S) reduce to $h(t)=g(t)$, which is trivially true. If $\sigma$ is a positive integer, (A.4) and (A.5) are again true, for in this case $h(t)=\left(d^{\sigma} / d t^{\sigma}\right)\left[t^{\circ} g(t)\right]$. The case $\sigma>0$, $\sigma$ not an integer, can be dealt with as follows: Define the function $\tilde{g}(t)$ by $t^{\sigma} g(t)=t^{\tilde{\sigma}} \tilde{g}(t)$, where $\tilde{\sigma}=\sigma-[\sigma]-1$. Then $-1<\tilde{\sigma}<0$ and $\tilde{g}(t)=t^{[\sigma]+1} g(t)$. Then as we have shown above, (A.4) and (A.5) hold with $\sigma$ and $g(t)$ replaced by $\tilde{\sigma}$ and $\tilde{g}(t)$, respectively. Using the definitions of $\tilde{\sigma}$ and $\tilde{g}(t)$, (A.4) and (A.5) can now be easily obtained. This completes the proof of the theorem. 


\section{References}

[1] D. Levin, "Development of non-linear transformations for improving convergence of sequences", Internat. J. Comput. Math. B3 (1973), 371-388.

[2] F. W. J. Olver, Asymptotics and special functions (Academic, New York, 1974).

[3] A. Sidi, Convergence properties of some non-linear sequence transformations", Math. Comp. 33 (1979), 315-326.

[4] A. Sidi, "Analysis of convergence of the $T$-transformation for power series", Math. Comp. 35 (1980), 833-850.

[5] A. Sidi, "Numerical quadrature and non-linear sequence transformations; unified rules for the efficient computation of integrals with end-point singularities", Math. Comp. 35 (1980), 851-874.

[6] A. Sidi, "Numerical quadrature rules for some infinite range integrals.", Math. Comp. 38 (1982), 127-142. 